\title{
Desigualdades nos salários dos trabalhadores gaúchos ${ }^{1}$
}

\author{
Izete Pengo Bagolin* \\ Valter José Stülp**
}

\begin{abstract}
RESUMO - Este trabalho visa identificar os fatores que influenciaram as variações ocorridas na desigualdade dos salários recebidos pelos trabalhadores formais no Rio Grande do Sul nos setores agrícola, indústria e serviços entre 1996 e 2005. Para isso foram utilizados os dados da RAIS, calculado o índice de Gini e estimadas regressões em Cross-section. Os resultados do setor agrícola mostram que ocorreu variação da desigualdade da renda e estas variações foram decorrência de alterações no $\mathrm{VAB}$, na escolaridade e realocação de trabalhadores nas diferentes classes de rendimentos. $\mathrm{Na}$ indústria ocorreu redução na desigualdade dos rendimentos do trabalho, e esta redução ocorreu ao longo de um período marcado por alterações e oscilações importantes na indústria e com um aumento do número de trabalhadores ocupados inferior ao crescimento da produção. Nos serviços, ocorreu aumento de $32 \%$ no emprego formal durante o período estudado. O aumento do número de pessoas ocupadas ocorreu nas faixas salariais mais baixas - até dois salários mínimos. As reduções ocorridas foram nas faixas acima de cinco salários mínimos. Em termos de qualificação é possível perceber que o número de trabalhadores com ensino fundamental incompleto se reduziu e o número de trabalhadores com ensino fundamental completo aumentou nos três setores.
\end{abstract}

Palavras chaves: Salário formal. Desigualdade. Municípios do Rio Grande do Sul.

\section{INTRODUÇÃO}

A desigualdade de renda no Brasil é um assunto que vem sendo explorado e debatido sob várias perspectivas. Sua gravidade manteve o Brasil entre as piores distribuições de renda do mundo. O Estado do Rio Grande do Sul (RS), apesar de apresentar indicadores sócio-econômicos superiores à média nacional, detêm indicadores de desigualdade significativos tanto em aspectos qualitativos quanto quantitativos, tais como: desigualdade de renda, expectativa de vida, nível de educação e mortalidade infantil (OLIVEIRA, 2001).

\footnotetext{
${ }^{1}$ Este artigo está baseado nos resultados de uma pesquisa mais ampla da qual resultaram três outros artigos. STÜLP, V. J. ; BAGOLIN, I. P. . Determinantes das variações na desigualdade dos rendimentos do mercado formal de trabalho na agricultura do Rio Grande do Sul. Revista de Economia (Curitiba), v. 35, p. 73-89, 2009 BAGOLIN, I. P. ; STÜLP, V. J. ; Scavoni, Leonardo. A dinâmica do emprego e da desigualdade na renda do trabalho nos diferentes setores da indústria gaúcha entre 1996 e 2005. Análise Econômica (UFRGS), 2009 (aguardando impressão)

STÜLP, V. J. ; BAGOLIN, I. P. ; Scavoni, Leonardo. A dinâmica do emprego e da desigualdade na renda do trabalho n os serviços do Rio Grande do Sul. Redes (Santa Cruz do Sul), 2009 (aguardando publicação)

* Professora Doutora do PPGE/PUCRS. Endereço Eletrônico: izete.bagolin@pucrs.br.

** Professor Doutor do PPGE/PUCRS. Endereço Eletrônico: vstulp@pucrs.br.
} 
Especificamente no aspecto desigualdade de renda, o Estado do RS apesar de apresentar índice de Gini inferior à média nacional, é mais desigual que outros estados de menor renda per capita como é o caso de Goiás, por exemplo.

Estudos que analisam a desigualdade de renda, suas causas, efeitos e alterações são diversos. Dentre eles, destacam-se estudos buscando relacionar a desigualdade de renda com o crescimento econômico, e dentre estes a chamada hipótese de Kuznets (1955) tem sido freqüentemente utilizada. A pergunta que Kuznets procurava responder com sua hipótese era: tomando-se a distribuição inicial histórica de ativos como dada, as desigualdades pioram ou melhoram ao longo do tempo? Como a renda afeta a desigualdade? Kuznets postulava que em economias com baixo nível de desenvolvimento, essencialmente agrícolas, a desigualdade é baixa, pois, quando existe pouco a ser distribuído, a concentração é baixa. Quando começa a ocorrer crescimento econômico e a economia passa para uma fase industrial as desigualdades aumentam e só irão diminuir quando uma fase avançada do processo de desenvolvimento for atingida. Seguindo essa ótica é de se esperar que, quando plotarmos no eixo horizontal a renda contra a desigualdade no eixo vertical, deve-se obter um U-invertido, o qual é conhecido na literatura como uma lei do desenvolvimento econômico. A literatura sobre desenvolvimento econômico apresenta vários estudos mostrando a aplicação prática da teoria de Kuznets, sendo que os resultados não são homogêneos.

Conforme Fields (2001), a intuição que está por trás da hipótese de Kuznets é que o crescimento econômico gera uma gradual realocação das atividades econômicas. Inicialmente ocorreria uma desigualdade relativamente baixa, quando o país possui atividades econômicas tradicionais, dirigindo-se para uma desigualdade relativamente alta quando se encontra numa economia moderna, de renda média, e voltando a apresentar baixa desigualdade quando atingir um estado avançado de desenvolvimento. Kuznets (1955) apud Fields (2001) produziu exemplos empíricos mostrando que a desigualdade iria primeiro aumentar no processo de mudança intersetorial e depois diminuir.

Analisando dados da economia doméstica, Bêrni ett alli (2002) testaram a hipótese de Kuznets para os municípios do Rio Grande do Sul através de um estudo cross-section, utilizando dados do ano de 1991. Os resultados sugerem uma aceitação cautelosa da hipótese de Kuznets. No caso da renda do setor agropecuário, os resultados mostram que à medida que a renda cresce, a desigualdade de renda vai diminuindo, até estabilizar num patamar pouco inferior a 0,6. Para este setor não foi observado padrão de Kuznets. Para o setor industrial e o 
de serviços, por outro lado, a hipótese de Kuznets não pode ser descartada, pois existem evidências que indicam a possível existência.

Analisando dados do crescimento da renda e desigualdade no Estado do Rio Grande do Sul, no período entre 1970 e 1991, Oliveira (2001) observou que no período 1970/80 ocorreu um aumento na renda total de 32,76\%, e no período total, 1970/91, o aumento foi de 24,55\%. Quando a renda per capita é analisada, observa-se uma queda generalizada no período 1980/91. Esta queda ocorreu devido à recessão pela qual o país passou na década de 80 e também pelo aumento da população em percentual superior ao aumento na renda.

Oliveira (2001) mostra que no período 1970/91, o RS apresentou aumento na desigualdade de renda. $\mathrm{O}$ autor decompôs esta desigualdade em desigualdade intermunicipal e desigualdade intramunicipal. $\mathrm{O}$ aumento na desigualdade de renda no Estado é explicado em sua maioria pelo aumento na desigualdade intramunicipal, sendo que o aumento na desigualdade intermunicipal é pouco expressivo.

A desigualdade de renda pode ser analisada utilizando subdivisões tais como rural e urbano, homens e mulheres, renda do trabalho, etc. Neste trabalho, o foco de interesse será o comportamento da desigualdade na renda do trabalho recebida pelos trabalhadores formais nos três setores de atividades - agricultura, indústria e serviços - do Rio Grande do Sul no período de 1996-2005.

Busca-se neste trabalho entender o comportamento da desigualdade sob duas perspectivas, ou seja: i) Identificar a existência de alterações na desigualdade salarial e, ii) Testar quais os fatores que foram os principais responsáveis por estas alterações entre os anos de 1996 e 2005. Sabe-se que o trabalho formal é apenas parte do trabalho que gera renda e que um número significativo de trabalhadores está no setor informal. A opção por trabalhar com o mercado formal de trabalho é decorrência do objetivo do trabalho se comparar com o comportamento da desigualdade ao longo do tempo e, por isso, é necessário ter dados oficiais para a renda do trabalho e também para outras variáveis de controle e/ou explicativas. Sendo assim, é impossível estimar as equações propostas para o trabalho como um todo ou mesmo para o mercado informal por não existirem dados disponíveis ao longo do tempo para todas as variáveis desejadas.

O estudo está organizado da seguinte forma: Além desta introdução, na parte dois são apresentados os procedimentos metodológicos. Na terceira parte os resultados das estimações e discussão. Por fim são apresentadas as considerações finais. 


\section{METODOLOGIA}

A análise consiste em avaliar o efeito de um conjunto de variáveis sobre a mudança na desigualdade da distribuição dos rendimentos recebidos pelos trabalhadores formais expressos em termos do salário formal, em nível municipal. A desigualdade na distribuição dos rendimentos é expressa em termos do índice de Gini. Calculam-se, ao nível dos municípios do Rio Grande do Sul, os índices de Gini das distribuições dos salários recebidos pelos trabalhadores referentes aos anos de 1996 e 2005. Os dados sobre o número de trabalhadores em cada faixa salarial expressa em termos de salários mínimos são do Ministério do Trabalho e Emprego (dados da RAIS). Para verificar a variação na desigualdade ao nível de cada município subtrai-se o valor do índice de Gini de 1996 do de 2005. O objetivo é buscar as causas dessa variação e, assim, a variável explicada é a variação no índice de Gini, entre estes dois anos, ou seja, a variação na desigualdade dos salários no período em estudo.

A análise é realizada através de uma regressão estatística, utilizando o método dos Mínimos Quadrados Ordinários em que a variável dependente é a variação ocorrida no índice de Gini em cada Município entre 1996 e 2005. A base de análise são os municípios do Rio Grande do Sul. Houve necessidade de agregar alguns municípios do ano de 2005, afim de que cada região se referisse à mesma área geográfica, tanto em 1996 como em 2005. Isto foi necessário devido às emancipações municipais ocorridas neste período, fazendo com que a área de um município existente em 1996 se referisse à área de mais de um município em 2005. O procedimento de agregação homogeneizou o número de observações nos dois períodos. Posteriormente foram estimadas regressões para cada setor.

Para o setor agrícola, a equação geral estimada tem o seguinte formato:

$\Delta G=\alpha+\beta$ pestmensos $20+\beta$ pestmais $200+\beta$ vanimanpeme $/$ vanimagra $+\beta$ vlavo $/$ vtotal + $\beta$ arealav / areapst $+\beta c v /$ halavo $+\beta$ taxcrescvab $+\beta$ mupart $96 a 051 a 1,5 \mathrm{sm}+$ ... $\ldots+$ Bmupart $96 a 05$ mais $20+\beta \Delta$ Gescola $+\mu$

Onde:

$\Delta G=$ variação do índice de Gini entre 1996 e 2005.

pestmenos 20 = percentual dos estabelecimentos agrícolas com menos de 20 hectares;

pestmais $200=$ percentual dos estabelecimentos agrícolas com mais de 200 hectares vanimapeme/vanimagra $=$ valor da produção dos animais de pequeno e médio porte em relação ao valor da produção dos animais de grande porte;

vlavo/vtotal = participação da lavoura no valor da produção agropecuária total; 
arealav/areapast $=$ área de lavoura em relação à área de pastagem.

$\mathrm{cv} /$ halavo $=$ expressa o grau de mecanização e é o número de cavalos vapor de trator existentes no município por hectare de lavoura.

txcrescvab = é a taxa de crescimento do VAB - valor adicionado da agropecuária $(\mathrm{VAB})$ do município entre 1996 e $2003 .^{2}$

mupart96a05trab1,0a1,5sm e mupart96a05trab1,5a2,0sm = são variações nos percentuais dos trabalhadores situados em cada faixa salarial entre 1996 e 2005.

$\Delta$ Gescola $=$ Gescola2005 - Gescola1996 = mudança, de 1996 a 2005, no índice de Gini da escolaridade entre os trabalhadores da agricultura.

$\mu=$ é termo de erro aleatório

No caso de setor industrial, buscando encontrar alguma explicação para a variação do índice de Gini da distribuição dos salários entre 1996 e 2005, estimou-se uma regressão estatística para cada setor industrial.

A variável explicada em cada regressão é a mudança ocorrida na distribuição dos salários, no período analisado, ou seja, o índice de Gini de 2005 menos o índice de Gini de 1996. As variáveis explicativas são as mudanças na participação dos trabalhadores do setor e do município nos níveis de escolaridade expressas em diferenças de percentuais; a variação percentual no salário médio ou per capita do trabalhador do setor e município de 1996 a 2005; a dimensão do setor no município, expressa em número de trabalhadores em 2005; a expansão do setor no município em termos de variação percentual do número de trabalhadores assalariados existentes no mesmo, de 1996 a 2005.

No setor serviços a variável explicada em cada regressão é a mudança ocorrida na distribuição dos salários, no período analisado, ou seja, o $\Delta \mathrm{Gini}^{3}$. As variáveis explicativas são: (i) as mudanças na participação dos trabalhadores do setor e do município nos níveis de escolaridade expressas em diferenças de percentuais. Assim, por exemplo, considera-se o percentual dos trabalhadores, dentre os do setor e do município, que, em 2005, tinham a $8^{\underline{a}}$ série incompleta, subtraindo-se deste percentual aquele referente aos trabalhadores, com as mesmas características, só que relativo ao ano de 1996. O mesmo procedimento foi aplicado a

\footnotetext{
2 A fonte de informação para a estimativa desta variável é a Fundação de Economia e Estatística (FEE) do Rio Grande do Sul. No período de 1996 a 1998 a FEE utilizou uma metodologia para o cálculo desta variável. A partir de 1999 a metodologia utilizada mudou. Assim, calcularam-se as taxas de crescimento do VAB de 1996 a 1998 e de 1999 a 2003 (último ano com a informação disponível). Considerou-se a soma das taxas destes dois períodos como uma variável explicativa. Esta soma exclui a taxa de crescimento do VAB de 1998 a 1999, pelo motivo da mudança na metodologia de cálculo. Foi utilizado o VAB real.

3 Índice de Gini de 2005 menos o índice de Gini de 1996.
} 
todos os níveis de escolaridade. Nas regressões somente foram incluídas as diferenças referentes aos níveis da $8^{\underline{a}}$ série incompleta até o superior. A inclusão de todos os níveis resultaria em multicolinearidade perfeita entre estas variáveis; (ii) mudança percentual no salário médio ou per capita do trabalhador do setor e município de 1996 a 2005; (iii) a dimensão do setor no município, expressa em número de trabalhadores em 2005; (iv) a expansão do setor no município em termos de variação percentual do número de trabalhadores assalariados, existentes no mesmo, de 1996 a 2005.

A seguir são apresentados os principais resultados para cada setor de atividade.

\section{RESULTADOS}

Para o setor agrícola os resultados mostram que a estrutura agrária do município influencia a desigualdade na distribuição dos rendimentos salariais. Municípios onde predominam estabelecimentos rurais com menos de 20 hectares ou então com mais de 200 hectares apresentam um aumento na desigualdade na distribuição dos salários agrícolas. Porém, o impacto dos grandes estabelecimentos sobre esta desigualdade é maior do que o efeito dos pequenos estabelecimentos. Quanto ao tipo de exploração, somente ocorre um efeito positivo sobre o índice de Gini quando aumenta a importância da criação de animais de pequeno e médio porte em relação aos de grande porte. A área de lavouras e a intensidade de sua mecanização não apresentaram impacto algum sobre o índice de Gini da distribuição dos salários.

Em relação à variação na participação dos trabalhadores assalariados nas diversas faixas salariais observa-se que o aumento no percentual de trabalhadores situados na faixa de um a dois salários mínimos diminui a desigualdade na distribuição dos rendimentos salariais. Já o aumento na participação dos trabalhadores nas faixas de três a quatro, de cinco a dez e mais de vinte salários mínimos eleva o valor do índice de Gini da desigualdade entre os rendimentos salariais.

A taxa de crescimento do VAB do setor agropecuário apresenta um efeito negativo estatisticamente significante sobre o índice de Gini da distribuição dos rendimentos salariais. O impacto da desigualdade na escolaridade sobre o índice de Gini da distribuição dos rendimentos salariais é positivo como é esperado. Como os rendimentos salariais dependem, em parte, da escolaridade do trabalhador, quanto mais desigual for o acesso a educação, mais desigual será a distribuição dos rendimentos salariais. 
A partir da queda dos percentuais de participação dos trabalhadores assalariados da agricultura do Rio Grande do Sul nos níveis de escolaridade extremos e aumento da sua participação nos níveis centrais, pode-se concluir que houve uma redução na desigualdade da escolaridade destes trabalhadores. Isto deve, portanto, ter contribuído para uma redução na desigualdade da distribuição dos rendimentos salariais, conforme o resultado da regressão.

No que tange ao setor industrial, em nove dos doze setores industriais estudados observa-se que o aumento percentual do salário per capita eleva o índice de Gini da distribuição dos salários, ou seja, torna-a mais desigual. Neste caso, um percentual significativo da indústria do Rio Grande do Sul estaria na parte crescente da curva de Kuznets.

Em três setores (alimentos e bebidas; calçados; e mecânica) quanto maior a indústria no município mais desigual é a distribuição dos salários. Isto poderia se explicar pelo aumento do número de cargos de chefia e de outros que exijam mais qualificação, gerando desigualdades nos salários, quando a fábrica se torna maior.

Examinando-se os impactos das mudanças ocorridas nos níveis de escolaridade dos trabalhadores sobre a distribuição nos seus níveis salariais verifica-se que os mesmos variam muito conforme o setor industrial. Esperava-se que níveis de escolaridade mais elevados estivessem associados com maiores níveis salariais. Assim, aumentos nas participações percentuais dos trabalhadores nos níveis mais elevados de escolaridade aumentariam o índice de Gini, pois estes trabalhadores receberiam salários mais altos que os da grande maioria. $\mathrm{O}$ oposto aconteceria com os aumentos nas participações dos trabalhadores nos níveis mais baixos de escolaridade. Em alguns setores industriais foi exatamente isto que aconteceu.

No setor de alimentos e bebidas o aumento na participação dos trabalhadores no nível de escolaridade superior incompleto aumentou o índice de Gini. No setor de borracha, fumo e couro uma maior participação dos trabalhadores com $8^{\text {a }}$ série incompleta reduz o índice de Gini. No setor de calçados uma maior participação dos trabalhadores nos níveis de $8^{\circ}$ série incompleta e de $2^{\circ}$ grau completo reduz o índice de Gini e nos níveis de superior incompleto e completo aumenta o índice de Gini.

Verifica-se que o impacto da maior participação de trabalhadores com curso superior completo e também com superior incompleto (exceção da indústria mecânica) nos setores onde foram estatisticamente significantes é no sentido do aumento do índice de Gini ou da desigualdade de salários. Porém, o sentido do impacto de uma maior participação dos trabalhadores com nível de escolaridade de $2^{\underline{\alpha}}$ grau completo ou menor varia conforme o 
setor industrial considerado. A tendência dos salários nos vários setores industriais é no sentido de uma redução, tendendo a grande maioria para a faixa de um a dois salários mínimos. Por isto se explica que os níveis de escolaridade mais elevados, com salários mais altos, aumentem o índice de Gini uma vez que estes salários diferem da tendência geral.

No setor dos serviços, de 1996 a 2005 praticamente não houve influência da escolaridade de $8^{\underline{a}}$ série incompleta ou completa sobre as mudanças no índice de Gini da distribuição dos salários. A única exceção é o impacto da escolaridade de $8^{\underline{a}}$ série incompleta que influencia positivamente a mudança no índice de Gini dos salários no setor de Alojamento, Alimentação e Mídia. O nível de escolaridade de $2^{\circ}$ grau incompleto influenciou positivamente a mudança no índice de Gini na Administração Pública, no Alojamento, Alimentação e Mídia e em Transportes e Comunicações.

O coeficiente do impacto da escolaridade de $2^{\underline{o}}$ grau completo sobre a mudança no índice de Gini, na Administração Pública, é negativo, apesar da redução do salário médio do trabalhador com esta escolaridade ter sido maior do que a do salário médio total do setor. $\mathrm{O}$ impacto da escolaridade de $2^{-}$grau completo sobre as mudanças no índice de Gini da distribuição de salários foi positivo na Administração Técnica Profissional, no Alojamento, Alimentação e Mídia e nos Transportes e Comunicações.

O nível de escolaridade superior incompleto influenciou positivamente a mudança no índice de Gini da distribuição de salários no setor de Alojamento, Alimentação e Mídia. A escolaridade correspondente ao nível superior completo influenciou positivamente as mudanças no índice de Gini em Alojamento, Alimentação e Mídia, no Comércio de Atacado, na Construção Civil e na Medicina, Odontologia e Veterinária. Por outro lado, o nível de escolaridade superior completo teve um efeito negativo sobre a mudança no índice de Gini em Serviços de Utilidade Pública, apesar de a variação do salário médio setorial ter sido de 2,21 salários mínimos (de 9,03 a 6,82) enquanto a do salário médio do trabalhador, com esta escolaridade, ter sido também maior, ou seja, 5,44 (de 16,26 a 10,82).

Constatou-se, portanto, que em geral, quando a queda no salário médio do trabalhador com uma dada escolaridade foi maior do que a redução salarial no total do setor ou ela foi até um patamar inferior ao do salário setorial médio, este nível de escolaridade exerceu um impacto positivo sobre o índice de Gini. Isto significa que ele contribuiu para uma maior desigualdade na distribuição salarial. Em caso contrário, o impacto da escolaridade foi negativo, ou seja, no sentido de redução da desigualdade salarial. Ocorreram duas exceções a esta explicação para o sentido dos impactos da escolaridade. 
Por sua vez, o efeito da variação percentual do salário per capita sobre a mudança do índice de Gini da distribuição dos salários foi negativo na Administração Pública, em Instituições Financeiras e em Serviços de Utilidade Pública. Estes eram os setores que, juntamente com o do Ensino, apresentavam em 2005 os maiores salários médios por trabalhador. Portanto, estes setores estariam na fase decrescente da Curva de Kuznets.

O impacto da variação percentual do salário per capita sobre a mudança do índice de Gini da distribuição dos salários foi positivo nos setores da Administração Técnica Profissional, do Alojamento, Alimentação e Mídia, do Comércio de Atacado, do Comércio de Varejo, da Construção Civil e da Medicina, Odontologia e Veterinária. Com exceção deste último setor, os demais apresentavam em 2005 os menores níveis de salário médio por trabalhador. Estes setores estariam na fase crescente da Curva de Kuznets.

Quanto ao número total de trabalhadores do setor empregados no município, em 2005, verifica-se que esta variável apresenta um impacto positivo sobre o índice de Gini da distribuição dos salários na Administração Pública, na Administração Técnica Profissional, no Alojamento, Alimentação e Mídia, no Comércio de Varejo, no Ensino, nas Instituições Financeiras e nos Serviços de Utilidade Pública. Nestes setores, quanto maior o número de trabalhadores empregados no município, mais desigual seria a distribuição dos salários. A explicação para isto poderia ser o fato de que cargos mais elevados, com remuneração mais alta, num determinado município, somente se justificariam quando o setor empregasse um número elevado de trabalhadores.

A expansão do setor em um determinado município, entre 1996 e 2005, expressa como a variação percentual do número de trabalhadores empregados, apresentou um impacto positivo sobre o índice de Gini nos setores de Alimentação, Alojamento e Mídia e no de Ensino. O impacto foi negativo em Transportes e Comunicações.

\section{CONCLUSÕES}

Os resultados evidenciam uma tendência de redução da desigualdade na distribuição dos rendimentos do trabalho formal dos trabalhadores do Rio Grande do Sul. Esta redução na desigualdade ocorreu ao longo de um período marcado por alterações e oscilações importantes na indústria e com aumento do número de trabalhadores inferior ao crescimento acumulado da produção. Ou seja, ocorreu aumento na produtividade do trabalho. Ao mesmo tempo ocorreu uma elevação do nível de escolaridade dos trabalhadores ocupados nos diferentes setores. 
O que mais chama atenção, porém, é que a redução na desigualdade é conseqüência de um achatamento salarial e do aumento do número de trabalhadores ocupados em empregos de menor remuneração. Em outras palavras, os trabalhadores estão mais qualificados, produzem mais e ganham menos.

A análise desenvolvida evidencia que:

(i) aumentou o número de trabalhadores recebendo até três salários mínimos, sendo este aumento mais significativo no grupo que recebe até dois salários mínimos;

(ii) o número de trabalhadores que recebiam acima de cinco salários mínimos diminuiu;

(iii) o nível médio de escolaridade dos trabalhadores gaúchos ocupados nos serviços aumentou entre os dois períodos;

(iv) houve redução no número de trabalhadores analfabetos e com ensino fundamental incompleto e também dos trabalhadores com ensino superior completo.

\section{REFERÊNCIAS}

$2^{\circ}$ ENCONTRO DE ECONOMIA GAUCHA, 2, 2004, Porto Alegre. Crescimento e Desigualdade no Rio Grande do Sul: Uma Revisao da Curva de Kuznets para os Municipios Gauchos (1970-1991). 2004.

$1^{\circ}$ ENCONTRO DE ECONOMIA GAÚCHA (EEG), 2002, Porto Alegre. A Desigualdade Económica do Rio Grande do Sul, primeiras investigações sobre a Curva de Kuznets.

FIEDS, G. S. Poverty, inequality and development. New York. Cambridge Press. 1980.

FIELDS, Gary S. Distribuition and Development: a new look at the developing world. MIT Press. Cambridge, Massachusetts London, England. 2001

HOFFMANN, Rodolfo. Estatística para Economistas. Biblioteca Pioneira de Ciências Sociais. São Paulo. SP. 1998.

KUZNETS, Simon. Economic Growth and Income Inequality. The American Economic Review, v. XLV, n. 01.1955

LLEDÓ, V. D. Distribuição de renda, crescimento endógeno e política fiscal: uma análise cross-section para os estados brasileiros. Texto Para Discussão n. 441. IPE. RJ.

OLIVEIRA, Júlio César de. Desenvolvimento Humano, Desigualdade de Renda e Pobreza nos Conselhos Regionais e Municípios do Rio Grande do Sul entre 1970 e 1991. Porto Alegre: PPGE/UFRGS. Tese de Doutorado, Economia, não publicada. 2001

RAY, Debraj. Development Economics. Princeton University Press. Princeton, New Jersey, United States of America. 1998

RAIS Registro Anual de Informações Sociais. Ministério do Trabalho e Emprego. Departamento de Emprego e Salário. Coordenação-Geral de Estatísticas do Trabalho. 